\title{
The foldability of cylindrical foldable structures based on rigid origami
}

\author{
Cai Jianguo ${ }^{1}$ \\ Key Lab. of C \& PC Structures of Ministry of Education, National Prestress Engineering \\ Research Center, Southeast University \\ Si Pai Lou 2\#, Nanjing, China \\ e-mail: j.cai@seu.edu.cn; caijg_ren@hotmail.com \\ ASME Member

\section{Zhang Yuting} \\ School of Civil Engineering, Southeast University, China \\ e-mail:472661724@qq.com \\ Xu Yixiang \\ Department of Civil Engineering, Strathclyde University, Glasgow, UK \\ e-mail: Yixiang.xu@strath.ac.uk
}

\section{Zhou Ya}

School of Civil Engineering, Southeast University, China

Current address: Wuxi Architectural Design \& Research Institute Co. Ltd, Wuxi, Jiangsu, China

e-mail: zhouya5166@126.com

\section{Feng Jian}

School of Civil Engineering, Southeast University, China

e-mail: fengjian@seu.edu.cn

\begin{abstract}
Foldable structures, a new kind of space structures developed in recent decades, can be deployed gradually to a working configuration, and also can be folded for transportation, thus have potentially broad application prospects in the fields of human life, military, aerospace, building structures and so on. Combined with the technology of origami folding, foldable structures derive more diversified models, and the foldable structures in cylindrical shape are mainly studied in this paper. Some researchers use the theory of quaternion representing spatial fixed-point rotation and construct the rotating vector model to obtain the quaternion rotation sequence method (QRS method) analyzing origami, but the method is very limited and not suitable for the cylindrical foldable structures. In order to solve the problem, a new method is developed, which combines the QRS method and the dual quaternion method. After analyzing the folding angle via the QRS method for multi-vertex crease system and calculating the coordinates of all vertices via the dual quaternion, the rigid foldability can be checked. Finally, two examples are carried out to confirm validity and versatility of the method.
\end{abstract}

\footnotetext{
${ }^{1}$ Corresponding author.
} 


\section{INTRODUCTION}

As an art of paper folding, origami has raised considerable research interest in mathematics. On the other hand, origami has been proved as a valuable tool to develop various engineering applications in numerous fields [1-5]. Most recent research on origami science mainly addresses the foldability issue, which is still regarded as intractable in many cases [6]. Rigid origami is a branch of origami whose facets and crease lines can be replaced by rigid panels and hinges. It stipulates that all panels bounded by creases in an origami pattern are not allowed to stretch or bend during folding, and thus all creases act as rotational hinges. Among recent research on rigid origami, a great majority focuses on the problem of rigid foldability [7]. Rigid foldability pays attention to the entire folding procedure and the method to assess the rigid foldability.

"Miura-ori" is an example of a famous pattern of rigid-foldability [8]. It is a wellknown rigid origami structure utilized in the packaging of deployable solar panels for use in space or in the folding of maps [9]. A mathematical model based on the Gaussian curvature theory of polygonal surfaces has been proposed to study rigid origami by Huffman and Miura [10-11]. Belcastro and Hull [12] proposed a matrix method for modelling origami configurations, which derived the necessary condition of the rigid origami patterns with either single or multiple vertices. In order to represent sequential rotations around creases, the affine mapping from two dimensions to three dimensions was considered to obtain the loop-closure equations. With the diagram method and numerical method, Watanabe and Kawaguchi [13] derived the necessary condition to judge the rigid foldability. Streinu and Whiteley [14] linked single-vertex rigid origami to spherical polygonal linkages, and proved that via spherical motions, some single vertex origami could be rigidly folded into other configurations continuously. It has been generalized to a family of rigid foldable origami with quadrilateral mesh [15]. By considering an origami carton as one planar four-bar loop and two spherical $4 R$ linkage loops, Wei and Dai [16] studied the geometry and kinematics of this origami carton. Based on the concept of an assembly of spherical linkages, Wang and Chen [17] proposed the mobile assembly of spherical $4 R$ linkages to study the Kokotsakis type of the rigid origami patterns. Moreover, Liu et al. $[18,19]$ also established the kinematic and geometric compatibility conditions of the mobile assemblies of spherical $4 R$ linkages to find the general condition of the rigid origami pattern for the deployable prismatic structures. Wu and You [20] proposed a new crease pattern that allows a tall box-shaped bag with a rectangular base to be rigidly folded flat. A rotating vector model was also proposed by $\mathrm{Wu}$ and You [7], which establishes the loop-closure conditions among a group of characteristic vectors. With the relationship between the single-vertex origami and the spherical linkage mechanism, the rotating vector model can conveniently and directly describe arbitrary three-dimensional configurations and can detect some selfintersection. Then a QRS method and a dual quaternion transformation sequence (DQTS) method were given to study the rigid foldability of single-vertex and multi-vertex origami, respectively.

However, many open problems still exist on rigid foldability. For example, no general approach to judging rigid foldability of deployable cylinder structures has been proposed. But there are lots of needs for such structures in space missions because of the severe limitation of launching volume [21]. 
In this paper, the rigid foldability of cylindrical origami has been studied. Firstly, the rotating vector model and the quaternion rotation sequence (QRS method) proposed by $\mathrm{Wu}$ and You [7], were introduced to study the rigid foldability of foldable cylinder structures. The limitation of this method was discussed. Then a new method, based on the dual quaternion was proposed. Finally, two examples are studied to confirm validity and versatility of the proposed method.

\section{FOLDABILITY OF A CYLINDRICAL ORIGAMI}

\section{The rotating vector model}

It is very convenient to express a single-vertex origami by the rotating vector model. Therefore, this section will briefly introduce the rotating vector model. More details about this model can be found in Ref. [7]. A four-fold single-vertex flat pattern is shown in Fig.1(a). The rigid panels are denoted by $B_{i}(i=1,2,3,4)$. The creases are denoted by $\mathrm{E}_{\mathrm{i}}(\mathrm{i}=1,2,3,4) . \alpha_{i, j}$ represents the layout angles from edge $\mathrm{E}_{\mathrm{i}}$ to $\mathrm{E}_{\mathrm{j}}(i=1,2,3,4, j=2,3,4$, 1). Figure1(b) shows a configuration during the folding process. The edge vector $e_{i}$ is along the crease $E_{i}$ and the normal vector $n_{i}$ is perpendicular to panel $B_{i}$.

Figure 2 shows the rotational relationships between the vectors, which can be illustrated by some sequential steps. The angle between normal vectors of adjacent panels that intersects at edge $E_{i}$ is denoted as $\gamma_{i}$. Starting with $n_{1}$ and $e_{1}$. $e_{i}$ rotates to $e_{i+1}$ around the axis along the vector $n_{i}$ by the angle $\alpha_{i, j}$ and $n_{i}$ rotates to $n_{i+1}$ around the axis along the vector $e_{i}$ by the angle $\gamma_{i}$. It should be noted that the last two steps return to the initial vectors $\mathrm{e}_{1}$ and $\mathrm{n}_{1}$, respectively. Thus a closed-loop rotation sequence can be obtained.

\section{The QRS method}

The QRS method means applying the quaternion rotation operator to the edge and normal vectors in origami patterns. Consider the vectors $\mathrm{e}_{1}=(1,0,0)$ and $\mathrm{n}_{1}=(0,0,1)$ in a threedimensional Cartesian coordinate frame. Now the folding angle between the adjacent panels, $t_{\mathrm{i}}$, is equal to $\pi-\gamma_{\mathrm{i}}$. The sequential steps shown in Fig. 2 can be expressed as:

(1) $e_{1} \stackrel{n_{1}, \alpha_{12}}{\longrightarrow} e_{2}$

$$
\begin{aligned}
& \tilde{q}^{n_{1}}=\left\{\cos \left(\frac{\alpha_{12}}{2}\right), \sin \left(\frac{\alpha_{12}}{2}\right) n_{1 x}, \sin \left(\frac{\alpha_{12}}{2}\right) n_{1 y}, \sin \left(\frac{\alpha_{12}}{2}\right) n_{1 z}\right\} \\
& \tilde{e}_{2}=\tilde{q}^{n_{1}} \tilde{e}_{1}\left(\tilde{q}^{n_{1}}\right)^{*}
\end{aligned}
$$

(2) $n_{1} \stackrel{e_{2}, \pi-t_{2}}{\longrightarrow} n_{2}$

$$
\begin{aligned}
& \tilde{q}^{e_{2}}=\left\{\cos \left(\frac{\pi-t_{2}}{2}\right), \sin \left(\frac{\pi-t_{2}}{2}\right) e_{2 x}, \sin \left(\frac{\pi-t_{2}}{2}\right) e_{2 y}, \sin \left(\frac{\pi-t_{2}}{2}\right) e_{2 z}\right\} \\
& \tilde{n}_{2}=\tilde{q}^{e_{2}} \tilde{n}_{1}\left(\tilde{q}^{e_{2}}\right)^{*}
\end{aligned}
$$

(3) $e_{2} \stackrel{n_{2}, \alpha_{23}}{\longrightarrow} e_{3}$

$$
\begin{aligned}
& \tilde{q}^{n_{2}}=\left\{\cos \left(\frac{\alpha_{23}}{2}\right), \sin \left(\frac{\alpha_{23}}{2}\right) n_{2 x}, \sin \left(\frac{\alpha_{23}}{2}\right) n_{2 y}, \sin \left(\frac{\alpha_{23}}{2}\right) n_{2 z}\right\} \\
& \tilde{e}_{3}=\tilde{q}^{n_{2}} \tilde{e}_{2}\left(\tilde{q}^{n_{2}}\right)^{*}
\end{aligned}
$$

(4) $n_{2} \stackrel{e_{3}, \pi-t_{3}}{\longrightarrow} n_{3}$ 
ASME Journal of Mechanical Design

$$
\begin{aligned}
& \tilde{q}^{e_{3}}=\left\{\cos \left(\frac{\pi-t_{3}}{2}\right), \sin \left(\frac{\pi-t_{3}}{2}\right) e_{3 x}, \sin \left(\frac{\pi-t_{3}}{2}\right) e_{3 y}, \sin \left(\frac{\pi-t_{3}}{2}\right) e_{3 z}\right\} \\
& \tilde{n}_{3}=\tilde{q}^{e_{3}} \tilde{n}_{2}\left(\tilde{q}^{e_{3}}\right)^{*}
\end{aligned}
$$

(5) $e_{3} \stackrel{n_{3}, \alpha_{34}}{\longrightarrow} e_{4}$

$$
\begin{aligned}
& \tilde{q}^{n_{3}}=\left\{\cos \left(\frac{\alpha_{34}}{2}\right), \sin \left(\frac{\alpha_{34}}{2}\right) n_{3 x}, \sin \left(\frac{\alpha_{34}}{2}\right) n_{3 y}, \sin \left(\frac{\alpha_{34}}{2}\right) n_{3 z}\right\} \\
& \tilde{e}_{4}=\tilde{q}^{n_{3}} \tilde{e}_{3}\left(\tilde{q}^{n_{3}}\right)^{*}
\end{aligned}
$$

(6) $n_{3} \stackrel{e_{4}, \pi-t_{4}}{\longrightarrow} n_{4}$

$$
\begin{aligned}
& \tilde{q}^{e_{4}}=\left\{\cos \left(\frac{\pi-t_{4}}{2}\right), \sin \left(\frac{\pi-t_{4}}{2}\right) e_{4 x}, \sin \left(\frac{\pi-t_{4}}{2}\right) e_{4 y}, \sin \left(\frac{\pi-t_{4}}{2}\right) e_{4 z}\right\} \\
& \tilde{n}_{4}=\tilde{q}^{e_{4}} \tilde{n}_{3}\left(\tilde{q}^{e_{4}}\right)^{*}
\end{aligned}
$$

(7) $e_{4} \stackrel{n_{4}, \alpha_{41}}{\longrightarrow} e_{5}$

$$
\begin{aligned}
& \tilde{q}^{n_{4}}=\left\{\cos \left(\frac{\alpha_{41}}{2}\right), \sin \left(\frac{\alpha_{41}}{2}\right) n_{4 x}, \sin \left(\frac{\alpha_{41}}{2}\right) n_{4 y}, \sin \left(\frac{\alpha_{41}}{2}\right) n_{4 z}\right\} \\
& \tilde{e}_{5}=\tilde{q}^{n_{4}} \tilde{e}_{4}\left(\tilde{q}^{n_{4}}\right)^{*}
\end{aligned}
$$

(8) $n_{4} \stackrel{e_{5}, \pi-t_{1}}{\longrightarrow} n_{5}$

$$
\begin{aligned}
& \tilde{q}^{e_{5}}=\left\{\cos \left(\frac{\pi-t_{1}}{2}\right), \sin \left(\frac{\pi-t_{1}}{2}\right) e_{5 x}, \sin \left(\frac{\pi-t_{1}}{2}\right) e_{5 y}, \sin \left(\frac{\pi-t_{1}}{2}\right) e_{5 z}\right\} \\
& \tilde{n}_{5}=\tilde{q}^{e_{5}} \tilde{n}_{4}\left(\tilde{q}^{e_{5}}\right)^{*}
\end{aligned}
$$

In these equations, $\tilde{e}_{i}$ and $\tilde{n}_{i}$ are the quaternion representations of vectors $e_{\mathrm{i}}$ and $n_{\mathrm{i}}$, the quaternion ( $)^{*}$ is the conjugate element of quaternion ( ).

It can be found that the vectors $e_{5}$ and $n_{5}$ should be the same as two initial vectors $e_{1}$ and $n_{1}$ based on the rotating vector model. So the loop-closure equation system can be derived as

$$
\left\{\begin{array}{l}
\tilde{e}_{5}=\left\{0, e_{5 x}, e_{5 y}, e_{5 z}\right\}=\tilde{e}_{1}=\left\{0, e_{1 x}, e_{1 y}, e_{1 z}\right\} \\
\tilde{n}_{5}=\left\{0, n_{5 x}, n_{5 y}, n_{5 z}\right\}=\tilde{n}_{1}=\left\{0, n_{1 x}, n_{1 y}, n_{1 z}\right\}
\end{array}\right.
$$

which leads to

$$
\left\{\begin{array}{l}
e_{5 x}=e_{1 x}=1 \\
e_{5 y}=e_{1 y}=0 \\
e_{5 z}=e_{1 z}=0 \\
n_{5 x}=n_{1 x}=0 \\
n_{5 y}=n_{1 y}=0 \\
n_{5 z}=n_{1 z}=1
\end{array}\right.
$$

Six simultaneous equations are obtained. For a given origami pattern, all $\alpha_{\mathrm{i}, \mathrm{j}}$ are known and therefore all the equations can be solved if the folding angle $t_{1}$ is specified.

\section{Application of the QRS method to a cylindrical origami}

For a four-fold single-vertex origami model, the QRS method is an useful method to describe the folding configuration of the model. However, for the multi-vertex origami, a decomposition strategy should be used [7]. A closed path encircling all vertices is firstly chosen. Therefore, the multi-vertex system can be seen as a combination of multiple single-vertex subsystems. Then we can analyze those subsystems sequentially along the closed path in one direction. The input parameter of each single-vertex system can be 
given from its previous subsystem. Finally, check whether the geometric parameter, such as the folding angle, common in the first and the last subsystems has the same values. If so, the origami pattern has the global compatibility. Wu and You [7] used this strategy to study the motion of some multi-vertex origami patterns. However, they did not try to study the rigid foldability of a cylindrical origami.

Figure 3 shows a modified Miura origami pattern. Four this pattern, it can have a flat configuration. With the QRS method, its rigid foldability can be shown. If we combine the vertices $\mathrm{V}_{12}$ together, this origami pattern forms a cylinder configuration as shown in Fig.4. For the cylindrical origami, it also folds rigidly with the QRS method. However, many researchers have stated the cylinder structure with Miura origami pattern cannot move rigidly. It can be found that the QRS method is not suitable for checking the rigid foldability of cylinder structures. It can be seen that when the origami pattern given in Fig. 3 forms a cylinder, an additional geometric constraint has to be added to the system. The constraint is that two vectors, $\mathrm{V}_{0}$ and $\mathrm{V}_{12}$, should coincide during the motion.

\section{THE PROPOSED METHOD FOR CHECKING RIGID FOLDABILITY}

This section discusses the dual quaternion method, which is proposed to check the foldability of the cylindrical origami. $\mathrm{Wu}$ and You also proposed a dual quaternion transformation sequence (DQTS) method. Compared with the QRS method, the DQTS method operates in a more global sense. However, it requires more inputs to solve the loop-closure equation system [7]. Moreover, when this method is used for the origami with many vertices, the number of loop-closure equations will be very high leading to numerical difficulty. Furthermore, the rigid foldability of cylinder structures was not investigated by them. In this paper, an improved method based on the dual quaternion method and the vertex coordinate transfer is proposed. Furthermore, we can simplify the dual quaternion operation using the known folding angle, which leads to a transfer from the parameter calculation to the numerical calculation. Then the coordinates of all vertices during the motion can be obtained. The relationship between folding angle and the vertex coordinate is used to check the rigid foldability of the cylindrical origami.

The dual quaternion is introduced firstly. A dual number $\bar{z}$ is defined as the sum of a scalar part and a dual part

$$
\bar{z}=a+\epsilon b
$$

where $a$ and $b$ are both real numbers, $\epsilon^{2}=0$ and $\epsilon \neq 0$.

Thus a dual quaternion $\hat{Q}$ can be defined as

$$
\hat{Q}=\tilde{q}_{1}+\tilde{q}_{2} \boldsymbol{\epsilon}=a_{1}+a_{2} \mathbf{i}+a_{3} \mathbf{j}+a_{4} \mathbf{k}+\left(a_{5}+a_{6} \mathbf{i}+a_{7} \mathbf{j}+a_{8} \mathbf{k}\right) \boldsymbol{\epsilon}
$$

where $a_{1}, a_{2}, a_{3}, a_{4}, a_{5}, a_{6}, a_{7}, a_{8}$ are real numbers, $\mathbf{i}, \mathbf{j}, \mathbf{k}$ are imaginary units. $\hat{Q}$ can also be expressed as

$$
\hat{Q}=\left\{a_{1}, a_{2}, a_{3}, a_{4}, a_{5}, a_{6}, a_{7}, a_{8}\right\}
$$

Normally, any rigid motion can be seen as screw motion. For the cylindrical origami considered in this paper, the motion can be seen as the fixed rotational motion and the vertices translational motion. For the fixed rotation motion, if the vector $r_{1}=\left(r_{1 x}, r_{1 y}, r_{1 z}\right)$ is rotated about the vector $\mathbf{z}, \hat{r}_{1}$ is the dual quaternion represent of vector $r_{1}$. Then the resultant vector $\hat{r}_{2}$ is 


$$
\hat{r}_{2}=\hat{Q} \hat{r}_{1} \hat{Q}^{*}
$$

where the unit dual quaternion operator $\hat{Q}$ and its conjugate elements can be represented as

$$
\begin{aligned}
\hat{Q} & =\left\{\cos \left(\frac{\alpha}{2}\right), \sin \left(\frac{\alpha}{2}\right) z_{x}, \sin \left(\frac{\alpha}{2}\right) z_{y}, \sin \left(\frac{\alpha}{2}\right) z_{z}, 0,0,0,0\right\} \\
\text { and } \hat{Q}^{*} & =\left\{\cos \left(\frac{\alpha}{2}\right),-\sin \left(\frac{\alpha}{2}\right) z_{x},-\sin \left(\frac{\alpha}{2}\right) z_{y},-\sin \left(\frac{\alpha}{2}\right) z_{z}, 0,0,0,0\right\} .
\end{aligned}
$$

If vertex $\mathrm{V}_{1}$ is translated to vertex $\mathrm{V}_{2}$ along vector $\boldsymbol{n}=\left(n_{x}, n_{y}, n_{z}\right)$, the resultant vertex $\mathrm{V}_{2}$ is then given as

$$
\hat{V}_{2}=\hat{Q} \hat{V}_{1} \hat{Q}
$$

where the unit dual quaternion operator $\hat{Q}=\left\{1,0,0,0,0, \frac{d}{2} n_{x}, \frac{d}{2} n_{y}, \frac{d}{2} n_{z}\right\}$ and $d=$ the distance of the translation from vertex $\mathrm{V}_{1}$ to vertex $\mathrm{V}_{2}$.

Figure 5 shows the serial numbers of the vertices and fold lines of the cylindrical origami. Assume the coordinate of Vertex $V_{1}$ is $(0,0,0)$, the $\mathrm{x}$-axis is along the vector $V_{1} e_{1}$, the z-axis is along the normal vector of rigid plate $V_{1} n_{1}$. Then the vertex coordinates can be obtained by following sequential steps. Firstly, the vector $V_{1} e_{1}$ of Vertex $V_{1}$ rotates to vector $V_{1} e_{2}$ about the vector $V_{1} n_{1}$. Then the vector $V_{1} n_{1}$ rotates to vector $V_{1} n_{2}$ about the vector $V_{1} e_{2}$. After that, vector $V_{1} e_{2}$ rotates to vector $V_{1} e_{3}$ about the vector $V_{1} n_{2}$. Then the vertex $V_{1}$ translates to vertex $V_{2}$ along the vector $V_{1} e_{3}$. Repeat the four steps until the vertex is translated from $V_{1}$ to $V_{0}$ and finally the whole closed-loop sequence is completed. The dual quaternion form of the vectors $V_{1} e_{1}, V_{1} n_{1}$, and $V_{1}$ can be given as

$$
\begin{aligned}
& V_{1} \hat{e}_{1}=\left\{1,0,0,0,0, V_{1} e_{1 x}, V_{1} e_{1 y}, V_{1} e_{1 z}\right\}=\{1,0,0,0,0,1,0,0\} \\
& V_{1} \hat{n}_{1}=\left\{1,0,0,0,0, V_{1} n_{1 x}, V_{1} n_{1 y}, V_{1} n_{1 z}\right\}=\{1,0,0,0,0,0,0,1\} \\
& \hat{V}_{1}=\left\{1,0,0,0,0, V_{1 x}, V_{1 y}, V_{1 z}\right\}=\{1,0,0,0,0,0,0,0\}
\end{aligned}
$$

Then the calculated steps of vertex coordinates can be given as $V_{1} e_{1} \stackrel{V r_{1}, V q_{12}}{\longrightarrow} V_{1} e_{2}:$

$$
\begin{aligned}
& \hat{Q}^{V^{n_{11}}}=\left\{\cos \left(\frac{V_{1} \alpha_{12}}{2}\right), \sin \left(\frac{V_{1} \alpha_{12}}{2}\right) V_{1} n_{1 x}, \sin \left(\frac{V_{1} \alpha_{12}}{2}\right) V_{1} n_{1 y}, \sin \left(\frac{V_{1} \alpha_{12}}{2}\right) V_{1} n_{1 z}, 0,0,0,0\right\} \\
& V_{1} \hat{e}_{2}=\hat{Q}^{V_{n} V_{1}} V_{1} \hat{e}_{1}\left(\hat{Q}^{V_{n_{n}}}\right)^{*} \\
& V_{1} n_{1} \stackrel{V_{1 e_{2}, \pi-V_{12}}}{\longrightarrow} V_{1} n_{2}: \\
& \hat{Q}^{V e_{2}}=\left\{\cos \left(\frac{\pi-V_{1} t_{2}}{2}\right), \sin \left(\frac{\pi-V_{1} t_{2}}{2}\right) V_{1} e_{2 x}, \sin \left(\frac{\pi-V_{1} t_{2}}{2}\right) V_{1} e_{2 y}, \sin \left(\frac{\pi-V_{1} t_{2}}{2}\right) V_{1} e_{2 z}, 0,0,0,0\right\} \\
& V_{1} \hat{n}_{2}=\hat{Q}^{V_{1 / 2}} V_{1} \hat{n}_{1}\left(\hat{Q}^{V_{1 e_{2}}}\right)^{*} \\
& V_{1} e_{2} \stackrel{V_{1} m_{2}, V V_{2}}{\longrightarrow} V_{1} e_{3}: \\
& \hat{Q}^{V^{\prime \prime 2}}=\left\{\cos \left(\frac{V_{1} \alpha_{23}}{2}\right), \sin \left(\frac{V_{1} \alpha_{23}}{2}\right) V_{1} n_{2 x}, \sin \left(\frac{V_{1} \alpha_{23}}{2}\right) V_{1} n_{2 y}, \sin \left(\frac{V_{1} \alpha_{23}}{2}\right) V_{1} n_{22}, 0,0,0,0\right\} \\
& V_{1} \hat{e}_{3}=\hat{Q}^{V_{m_{2}}} V_{1} \hat{e}_{2}\left(\hat{Q}^{V_{r_{2}}}\right)^{*}
\end{aligned}
$$

Vector transformation:

$$
\begin{aligned}
& V_{2} \hat{e}_{1}=\left\{V_{1} \hat{e}_{3}(1), V_{1} \hat{e}_{3}(2), V_{1} \hat{e}_{3}(3), V_{1} \hat{e}_{3}(4), V_{1} \hat{e}_{3}(5),-V_{1} \hat{e}_{3}(6),-V_{1} \hat{e}_{3}(7),-V_{1} \hat{e}_{3}(8),\right\} \\
& V_{2} \hat{n}_{1}=V_{1} \hat{n}_{2}
\end{aligned}
$$

From Vertex $\mathrm{P}_{1}$ to vertex $\mathrm{P}_{2}$ by the distance $d_{1}$ : 


$$
\begin{aligned}
& \hat{Q} V_{1} V_{2}=\left\{1,0,0,0,0, \frac{d_{1}}{2} V_{1} e_{3 x}, \frac{d_{1}}{2} V_{1} e_{3,}, \frac{d_{1}}{2} V_{1} e_{33}\right\} \\
& \hat{V}_{2}=\hat{Q} V_{1} V_{2}\left(\hat{V}_{1}\right) \hat{Q} V_{1} V_{2}
\end{aligned}
$$

For the cylindrical origami pattern shown in Fig. 3, repeating Eqs. (19) to (23), we can obtain the coordinates of vertex $\hat{V}_{0}$. The sixth, seventh and eighth elements of this dual quaternion are the $\mathrm{x}, \mathrm{y}, \mathrm{z}$ coordinates of Vertex 0 . With the help of MATLAB, the coordinates of Vertex 0 with different folding angles are given in Table 1 and Fig. 6. It can be found the coordinates of Vertex 0 varied during the motion of the cylinder. That is to say, the cylindrical origami with Miura pattern is not rigidly foldable.

Rigid origami stipulates that all panels bounded by creases in an origami pattern are not allowed to stretch or bend during folding. The proposed method can also be used to check the foldability of multi-vertex cylindrical origami. The criterion is summarized as follows: (1)The folding angle can be solved by the QRS method. If the last crease vectors are same as the initial crease vectors after a closed-loop rotation but the folding angle in different folding configuration does not always remain the same as the original value, then the model does not have rigid foldability. Otherwise, go to step 2. (2)After obtaining the coordinates of the vertices using the dual quaternion method, if the coordinate of last vertex $V_{\mathrm{n}+1}\left(\right.$ or $\left.V_{0}\right)$ is not same as that of origin vertex $V_{1}$, then the cylinder does not have rigid foldability. (3)The coordinate curves of all vertices should keep continuous and smooth for a rigidly foldable cylinder.

\section{NUMERICAL EXAMPLES}

To confirm validity and versatility of the proposed method, foldability analyses of several types of crease patterns are carried out.

\section{Tachi cylindrical origami}

Figure 7(a) shows the formation of Tachi cylindrical origami [22]. Mirror the Miura-ori and then a Tachi origami is obtained. Figure 7(b) shows a typical unit of Tachi cylinder. The Tachi cylinder is rigidly foldable and has a single degree of freedom. Besides it has both flat configurations in fully folded state and fully unfolded state.

After analyzing the typical unit, it can be obtained that $\mathrm{P}_{1}$ and $\mathrm{P}_{3}$ are two-dimensional single-vertices, $\mathrm{P}_{2}$ and $\mathrm{P}_{4}$ are three-dimensional vertices which cannot fold flatly. Assuming the folding angle $V_{1} t_{1}$ of crease $V_{1} e_{1}$ as the input angle, all other corresponding folding angles of vertex $\mathrm{P}_{1}$ can be obtained. After vertex $\mathrm{P}_{1}$ is translated to $\mathrm{P}_{2}$ along the vector $V_{1} e_{3}$, all the folding angles of vertex $\mathrm{P}_{2}$ can be calculated. Following that analogy, the folding angles of vertices $\mathrm{P}_{3}$ and $\mathrm{P}_{4}$ can be obtained. The crease vector $V_{4} e_{3}$ should be the same as the crease vector $V_{1} e_{1}$. Therefore, if $V_{4} t_{3} \equiv V_{1} t_{1}$, the angle relationship satisfies the necessary condition of rigid foldability.

For the Tachi typical unit, the geometric parameters can be assumed as

$$
\begin{aligned}
& V_{1} \alpha_{12}=V_{1} \alpha_{41}=V_{3} \alpha_{23}=V_{3} \alpha_{34}=3 \pi / 4, \quad V_{1} \alpha_{23}=V_{1} \alpha_{34}=V_{3} \alpha_{12}=V_{3} \alpha_{41}=\pi / 4, \\
& V_{2} \alpha_{12}=V_{2} \alpha_{23}=V_{2} \alpha_{34}=V_{2} \alpha_{41}=3 \pi / 4, \quad V_{4} \alpha_{12}=V_{4} \alpha_{23}=V_{4} \alpha_{34}=V_{4} \alpha_{41}=\pi / 4 .
\end{aligned}
$$


The distance between two adjacent vertices is $d_{k}=1$. Then the folding angles are listed in Table 2.

It can be found from Table 2 that $V_{4} t_{3} \equiv V_{1} t_{1}$, which means the angle relationship satisfies the necessary conditions for rigid foldability. Then we will study the vertex coordinates relationship. Assuming the coordinate of $\mathrm{P}_{1}$ is $(0,0,0)$, the crease vector $V_{1} e_{1}=(1,0,0)$, and the normal vector of the rigid plate $V_{1} n_{1}=(0,0,1)$, with the dual quaternion method, the coordinates changes of the vertices are given in Table 3.

It can be found from Table 3 that the coordinate of $\mathrm{P}_{5}$ is the same as that of $\mathrm{P}_{1}$ during the motion, which indicates that the coordinate relationship satisfies the necessary conditions for rigid foldability. The coordinate changes of $\mathrm{P}_{1} \sim \mathrm{P}_{4}$ are shown in Figure 8. The changing curves are continuous and smooth, satisfying the last necessary condition for rigid foldability. Therefore, the Tachi cylindrical origami has a rigid foldability.

\section{Deployable cylinder}

Based on the concept of the Tachi-Miura Polyhedron [23], a new type of deployable cylinders was given in Fig. 9. The cylinder is formed by two pieces of Miura origami, which is connected by two six-creases origami patterns. Therefore, as shown in Fig.10, the Vertices 1 and 6 have six creases, and all other vertices have four creaes.

Assuming the folding angle $V_{1} t_{1}$ of crease $V_{1} e_{1}$ as the input angle, the other corresponding folding angles of vertex $\mathrm{P}_{1}$ can be calculated. After vertex $\mathrm{P}_{1}$ is translated to $\mathrm{P}_{2}$ along the vector $V_{1} e_{4}$, the folding angles of vertex $\mathrm{P}_{2}$ can be obtained. Following that analogy, the crease angle of vertex $\mathrm{P}_{8}$ can be obtained and the crease vector $V_{8} e_{3}$ should be the same as the crease vector $V_{1} e_{1}$. If $V_{8} t_{3} \equiv V_{1} t_{1}$, the angle relationship satisfies the necessary conditions for rigid foldability.

For the deployable cylinder, the geometry parameters can be assumed as

$$
\begin{gathered}
V_{1} \alpha_{12}=V_{1} \alpha_{23}=V_{1} \alpha_{56}=V_{1} \alpha_{61}=\pi / 4, V_{1} \alpha_{34}=V_{1} \alpha_{45}=\pi / 2 \\
V_{k} \alpha_{12}=V_{k} \alpha_{41}=\pi / 4, V_{k} \alpha_{23}=V_{k} \alpha_{34}=3 \pi / 4 \\
V_{k} \alpha_{12}=V_{k} \alpha_{41}=3 \pi / 4, V_{k} \alpha_{23}=V_{k} \alpha_{34}=\pi / 4 \\
V_{6} \alpha_{12}=V_{6} \alpha_{61}=\pi / 2, V_{6} \alpha_{23}=V_{6} \alpha_{34}=V_{6} \alpha_{45}=V_{6} \alpha_{56}=\pi / 4
\end{gathered}
$$

The distances between two adjacent vertices are $d_{1}=d_{5}=1, d_{2}=d_{3}=d_{4}=d_{6}=d_{8}=2, d_{7}=4$. The results are listed in Table 4.

It can be found that $V_{8} t_{3} \equiv V_{1} t_{1}$, so the angle relationship satisfies the necessary conditions for rigid foldability. However, $V_{1} t_{3}=V_{1} t_{5}=V_{6} t_{2}=V_{6} t_{6}=2 \pi$, indicating that the corresponding creases don't deploy during the motion.

Then we will study the vertex coordinates relationship. Assuming the coordinate of $\mathrm{P}_{1}$ is $(0,0,0)$, the crease vector $V_{1} e_{1}=(1,0,0)$, and the normal vector of the rigid plate $V_{1} n_{1}=(0,0,1)$, with the dual quaternion method, the changes in vertices coordinates are given in Table 5 .

It can be found that during the motion, the coordinate of $\mathrm{P}_{9}$ is the same as that of $\mathrm{P}_{1}$, indicating that the coordinate relationship satisfies the necessary conditions for rigid foldability. 
The changes of coordinate $\mathrm{P}_{1} \sim \mathrm{P}_{8}$ are shown in Fig. 11. The curves are continuous and smooth, satisfying the last necessary condition for rigid foldability. Therefore, the deployable cylinder can be proved rigidly foldable via the proposed method.

\section{CONCLUSIONS}

In this paper, we established a new method to study the rigid foldability of the multivertex cylindrical-shaped origami patterns. After analyzing the folding angles via the QRS method for multi-vertex crease origami, calculating the coordinates of all vertices using the dual quaternion method, the rigid foldability can then be checked. Compared with the original QRS method proposed by Wu and You [7], the method is able to check the rigid foldability of multi-vertex cylindrical origami. Through two examples, we demonstrated that the proposed method can analyze the folding angles and coordinates of vertices, and thereby predict its rigid foldability. However, due to the complexity of both the folding angles and vertex coordinates of the multi-vertex origami, we have yet to use the proposed method to develop an algorithm for detecting more cylindrical origami with rigid foldability, which will be the further study of this topic.

\section{ACKNOWLEDGMENTS}

The work presented in this article was supported by the National Natural Science Foundation of China (Grant No. 51308106 and No. 51278116), the Natural Science Foundation of Jiangsu Province (Grant No. BK20130614), the Specialized Research Fund for the Doctoral Program of Higher Education (Grant No. 20130092120018), a Project Funded by the Priority Academic Program Development of Jiangsu Higher Education Institutions and the Excellent Young Teachers Program of Southeast University. Authors thank all the anonymous reviewers for their valuable comments and thoughtful suggestions which improved the quality of the presented work. 


\section{REFERENCES}

[1] Sorguc A G, Hagiwara I, Selcuk S A. Origami in Architecture: A Medium of Inquiry for Design in Architecture. Metu Jfa, 2009, 26(2): 235-247.

[2] Hagiwara I. From Origami to Origamics. Science Japan Journal, 2008, July, 22-25.

[3] Guest S D. Deployable structures: Concepts and Analysis. PhD thesis, Cambridge University, Cambridge, UK, 1996.

[4] Ma J, You Z. Energy Absorption of Thin-Walled Square Tubes with a Prefolded Origami Pattern-Part I: Geometry and Numerical Simulation. Journal of Applied Mechanics-Transactions of the ASME, 2014, 81(1): 011003

[5] Ma J, You Z. Energy absorption of thin-walled beams with a pre-folded origami pattern. Thin-walled Structures, 2013, 73: 198-206

[6] Demaine E D, O'Rourke, J. A survey of folding and unfolding in computational geometry. In Mathematical sciences research institute publications, 2005, vol. 52, pp. 167-211. Berkeley, CA: MSRI Publications.

[7] $\mathrm{Wu} \mathrm{WN}$, You Z. Modelling rigid origami with quaternions and dual quaternions, Proceedings of the Royal Society A: Mathematical, Physical and Engineering Sciences, 2010, 466: 2155-2174

[8] Miura K. Map Fold a La Miura Style, Its Physical Characteristics and Application to the Space Science. In Research of Pattern Formation, edited by R. Takaki, pp. 77-90. Tokyo: KTK Scientific Publishers, 1989.

[9] Miura K. Method of packaging and deployment of large membranes in space, In: Proceedings of 31 stCongress of International Astronautics Federation (IAF-80-A31), Tokyo, Japan, 1980, 1-10.

[10] Huffman D. 1976 Curvature and crease: a primer on paper. IEEE Trans. Comput. C25, 1010-1019.(doi:10.1109/TC.1976.1674542)

[11] Miura K. 1989 A Note on Intrinsic Geometry of Origami. In Proc. 1st Int. Meet. of Origami Science and Technology, 6-7 December, Ferrara, Italy (ed. H. Huzita), pp. 239-249.

[12]Belcastro, S. \& Hull, T. 2002, Modeling the folding of paper into three dimensions using affine transformations. Linear Algebra Appl. 348, 273-282.

[13] Watanabe N, Kawaguchi K. 2006, The method for judging rigid foldability. In Origami 4Proc. 4th Int. Conf. on Origami in Science, Mathematics, and Education, Pasadena, CA, September 8-10, pp. 165-174. Natick, MA: AK Peters

[14] Streinu I, Whiteley W. 2005 Single-vertex origami and spherical expansive motions. Lecture Notes in Computer Science, no. 3742, pp. 161-173. Berlin, Germany: Springer.

[15] Tachi T. Generalization of Rigid Foldable Quadrilateral Mesh Origami. Proceedings of the International Association for Shell and Spatial Structures Symposium 2009, edited by Albert Domingo and Carlos Lazaro, pp. 2287-2294. Madrid: IASS, 2009.

[16] Wei GW, Dai JS. Origami-Inspired Integrated Planar-Spherical Overconstrained Mechanisms, J. Mech. Des., 2014, 136(5), 051003

[17] Wang KF, Chen Y. Rigid Origami to Fold a Flat Paper into a Patterned Cylinder, The 5th international conference on origami in science, Singapore, 2010.

[18] Liu SC, Chen Y, Lu GX. The rigid origami patterns for flat surface, Proceedings of the ASME 2013 International Design Engineering Technical Conferences and 
Computers and Information in Engineering Conference, IDETC/CIE 2013, August 47, 2013, Portland, Oregon, USA

[19]Liu SC, Lv WL, Chen Y, Lu GX. Deployable Prismatic Structures with Origami Patterns, Proceedings of the ASME 2014 International Design Engineering Technical Conferences \& Computers and Information in Engineering Conference, IDETC/CIE 2014 August 17-20, 2014, Buffalo, New York, USA

[20] Wu W, You Z. A solution for folding rigid tall shopping bags, Proc. R. Soc. London, Ser. A, 2011, 467, 2561-2574.

[21] Miura K, Tachi T, Miyazaki Y. Foldable space structures-foldable cylindrical polyhedron and IKAROS solar sail, IASS-APCS 2012 Symposium, May 21-24, 2012, Seoul, Korea

[22] Tachi T. One-DOF Cylindrical Deployable Structures with Rigid Quadrilateral Panels, in: A. Domingo, C. Lazaro (Eds.) The International Association for Shell and Spatial Structures Symposium 2009, Madrid: IASS, 2009, pp. 2295-2305.

[23] Miura K, Tachi T. Synthesis of rigid-foldable cylindrical polyhedral, Symmetry: Art and Science, Gmuend, Austria, 2010 
ASME Journal of Mechanical Design

\section{Figure Captions List}

Fig. 1 Origami crease pattern (a) The initially flat state (b) a folding configuration

Fig. 2 The closed-loop rotation sequence of the characteristic vectors decomposed into eight steps in the rotating vector model of the singlevertex fourfold system

Fig. 3

The modified Miura origami pattern

Fig. 4

The cylinder configuration

Fig. 5

The serial numbers of the vertex and fold lines

Fig. 6

The coordinates of Vertex 0

Fig. 7 Tachi origami model

Fig. 8 The coordinate changes of $\mathrm{P}_{1} \sim \mathrm{P}_{4}$

Fig. 9 Crease pattern of a deployable cylinder

Fig. 10 Vertices of the deployable cylinder (a) Vertex $\mathrm{P}_{1}$ (b) Vertex $\mathrm{P}_{2} \sim \mathrm{P}_{5}$ (c) Vertex $\mathrm{P}_{6}$ (d) Vertex $\mathrm{P}_{7} \sim \mathrm{P}_{8}$

Fig. 11 The coordinate changes of $\mathrm{P}_{1} \sim \mathrm{P}_{8}$ 
ASME Journal of Mechanical Design

\section{Table Caption List}

Table 1 The coordinates of Vertex 0

Table 2 The folding angles of Tachi typical unit

Table 3

The coordinates changes of the vertices

Table 4

The folding angles of Deployable cylinder

Table 5 The coordinates changes of the vertices 
ASME Journal of Mechanical Design

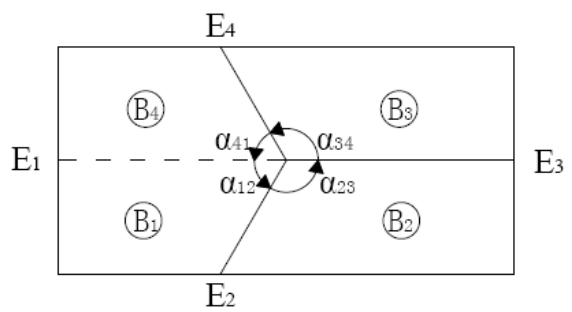

(a) The initially flat state

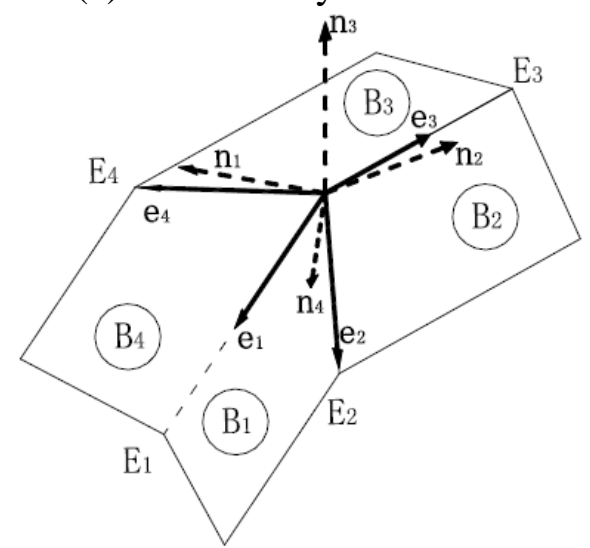

(b)a folding configuration

Fig.1 Origami crease pattern

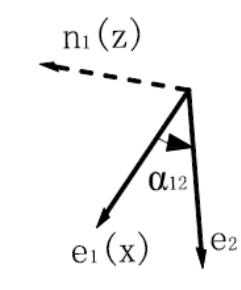

(1)

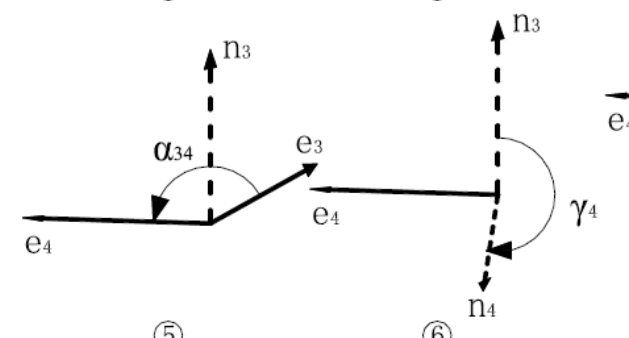

(6)

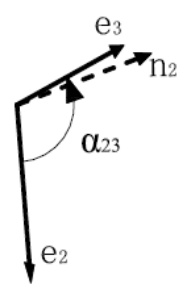

(3)

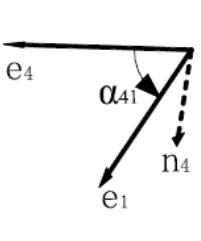

(7)

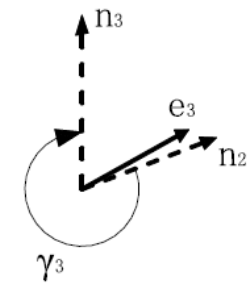

(4)

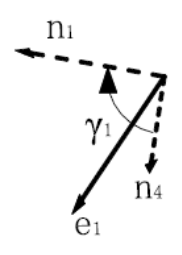

(8)

Fig. 2 The closed-loop rotation sequence of the characteristic vectors decomposed into eight steps in the rotating vector model of the single-vertex fourfold system. 


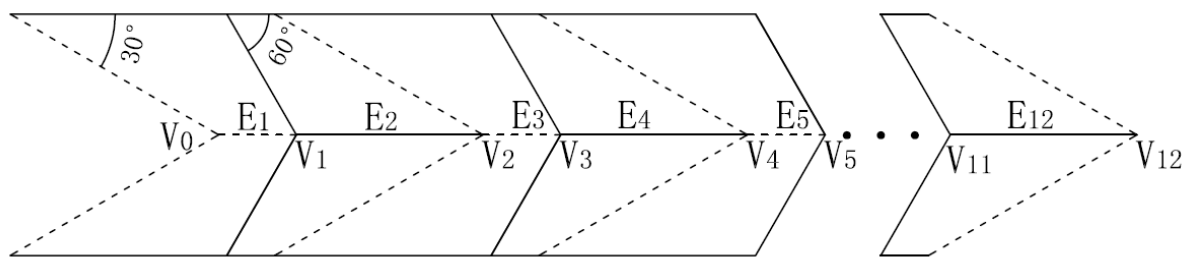

Fig.3 The modified Miura origami pattern

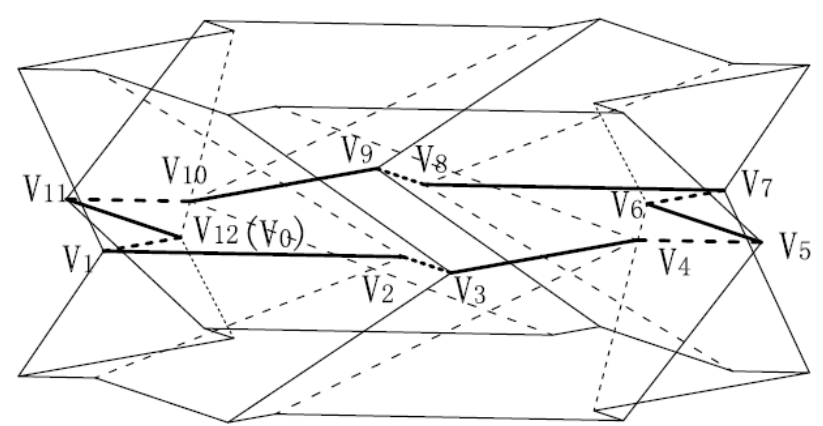

Fig.4 The cylinder configuration

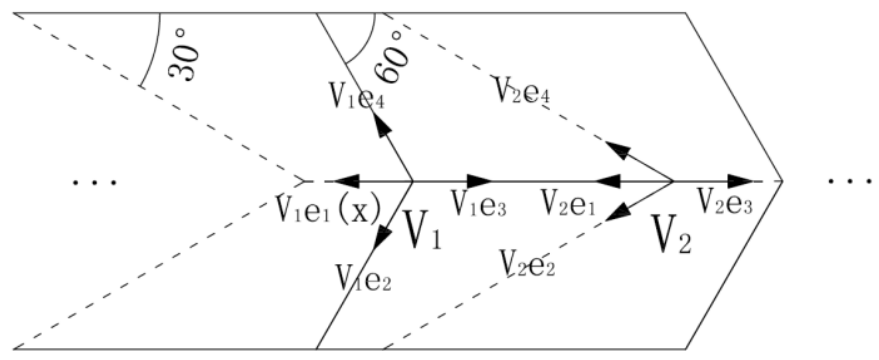

Fig. 5 The serial numbers of the vertex and fold lines

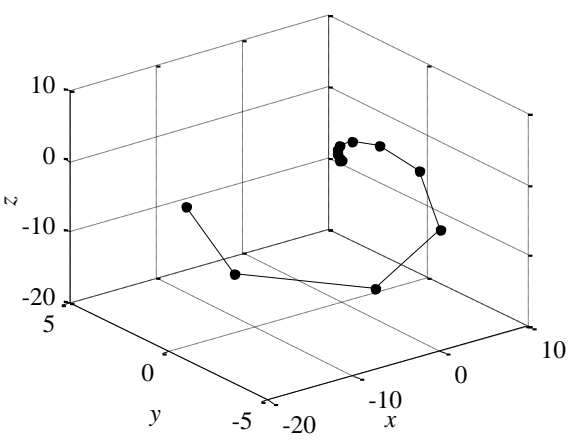

Fig. 6 The coordinates of Vertex 0 


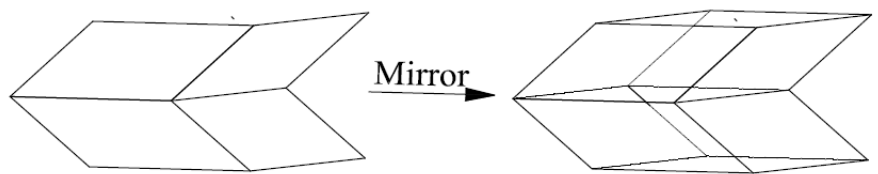

(a)Formation of Tachi origami

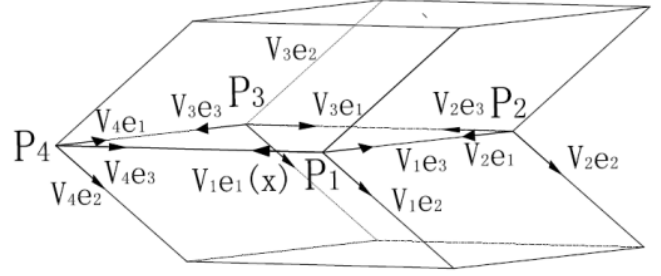

(b)A typical unit

Fig.7 Tachi origami model

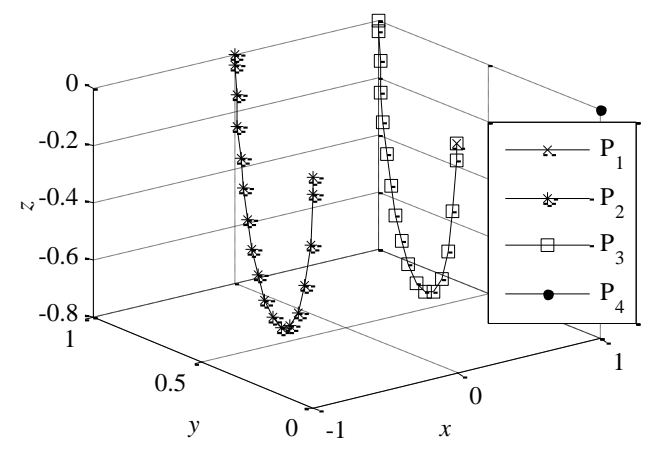

Figure 8 The coordinate changes of $\mathrm{P}_{1} \sim \mathrm{P}_{4}$

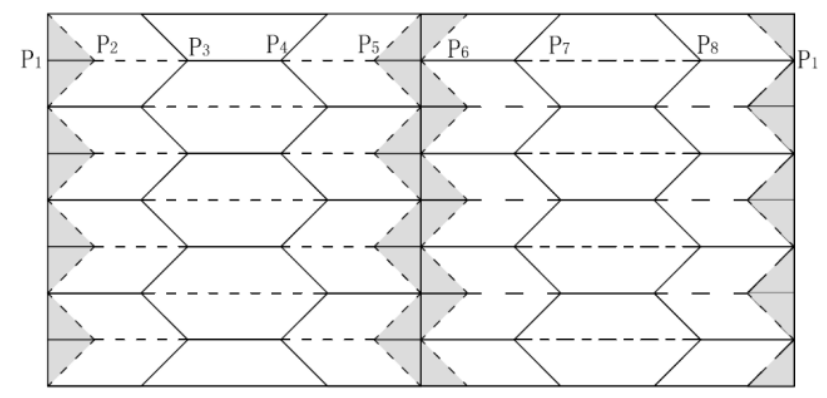

Figure 9 Crease pattern of a deployable cylinder 
ASME Journal of Mechanical Design

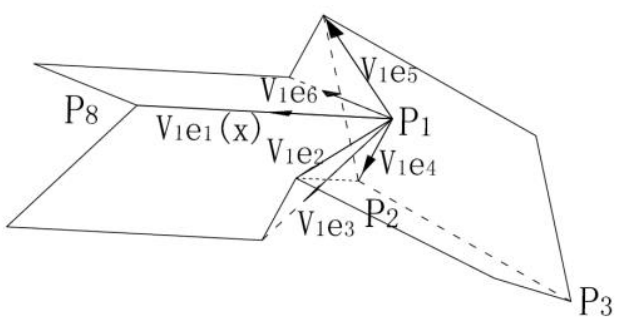

(a)Vertex $\mathrm{P}_{1}$

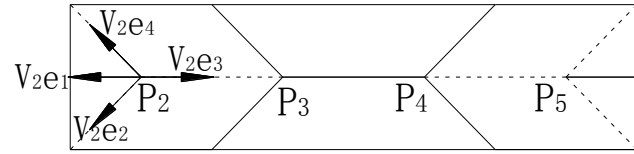

(b)Vertex $\mathrm{P}_{2} \sim \mathrm{P}_{5}$

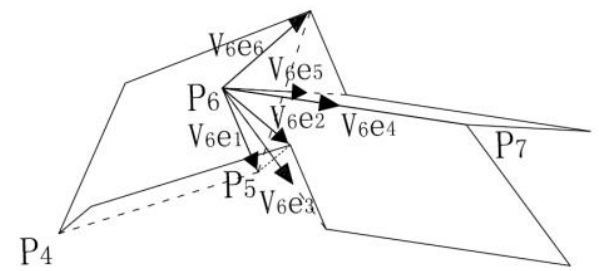

(c) Vertex $\mathrm{P}_{6}$

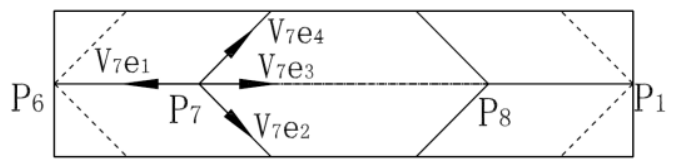

(d)Vertex $\mathrm{P}_{7} \sim \mathrm{P}_{8}$

Fig. 10 Vertices of the deployable cylinder

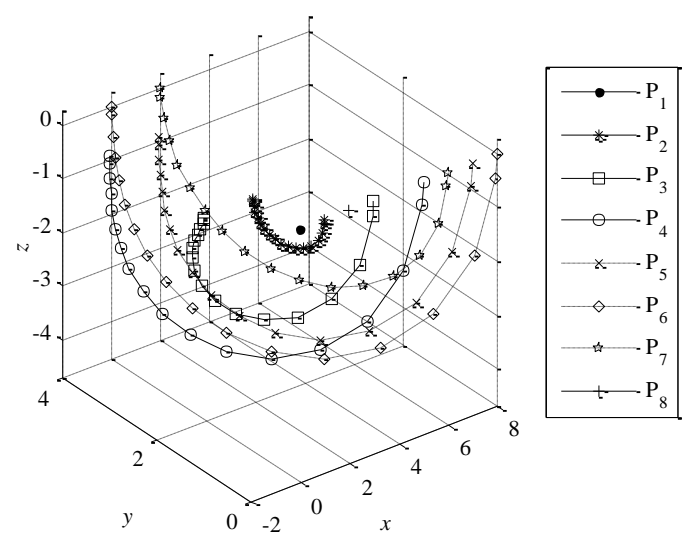

Fig. 11The coordinate changes of $\mathrm{P}_{1} \sim \mathrm{P}_{8}$ 
ASME Journal of Mechanical Design

Table 1 The coordinates of Vertex 0

\begin{tabular}{cccccc}
\hline$V_{1} t_{1}(\mathrm{rad})$ & 0 & 0.4 & 0.8 & 1.2 & 1.6 \\
\hline$V_{0} P_{x}$ & 0 & 0.001 & 0.0181 & 0.1264 & 0.6342 \\
$V_{0} P_{y}$ & 0 & 0.0028 & 0.0404 & 0.1911 & 0.5019 \\
$V_{0} P_{z}$ & 0 & -0.0001 & 0.0169 & 0.1301 & 0.5161 \\
\hline$V_{1} t_{1}(\mathrm{rad})$ & 2 & 2.4 & 2.8 & $\pi$ & -18 \\
\hline$V_{0} P_{x}$ & 2.5942 & 6.6825 & -1.737 & 0 & \\
$V_{0} P_{y}$ & 0.6148 & -0.9844 & -2.4346 & 0 & \\
$V_{0} P_{z}$ & 0.9588 & -2.5308 & -14.1109 & &
\end{tabular}


ASME Journal of Mechanical Design

Table 2 The folding angles of Tachi typical unit

\begin{tabular}{|c|c|c|c|c|c|c|c|c|c|}
\hline $\begin{array}{c}V_{1} t_{1}( \\
\text { Mountain) }\end{array}$ & $\pi$ & 3.4 & 3.8 & 4.2 & 4.6 & 5 & 5.4 & 5.8 & $2 \pi$ \\
\hline $\begin{array}{c}V_{1} t_{2}( \\
\text { Mountain) }\end{array}$ & 3.1416 & 3.505 & 4.0417 & 4.5237 & 4.9442 & 5.3112 & 5.638 & 5.9382 & 6.2832 \\
\hline $\begin{array}{c}V_{2} t_{2}( \\
\text { Mountain })\end{array}$ & 6.2832 & 5.9198 & 5.383 & 4.9011 & 4.4805 & 4.1135 & 3.7868 & 3.4866 & 3.1416 \\
\hline $\begin{array}{c}V_{3} t_{2}( \\
\text { Mountain) }\end{array}$ & 3.1416 & 3.505 & 4.0417 & 4.5237 & 4.9442 & 5.3112 & 5.638 & 5.9382 & 6.2832 \\
\hline $\begin{array}{c}V_{4} t_{2}( \\
\text { Mountain) }\end{array}$ & 6.2832 & 5.9198 & 5.383 & 4.9011 & 4.4805 & 4.1135 & 3.7868 & 3.4866 & 3.1416 \\
\hline $\begin{array}{c}V_{4} t_{3}( \\
\text { Mountain) }\end{array}$ & $\pi$ & 3.4 & 3.8 & 4.2 & 4.6 & 5 & 5.4 & 5.8 & $2 \pi$ \\
\hline
\end{tabular}

Table 3 The coordinates changes of the vertices

\begin{tabular}{cccccccccc}
\hline$V_{1} t_{1}(\mathrm{rad})$ & $\pi$ & 3.4 & 3.8 & 4.2 & 4.6 & 5 & 5.4 & 5.8 & $2 \pi$ \\
\hline$V_{1} P_{x}$ & 0 & 0 & 0 & 0 & 0 & 0 & 0 & 0 & 0 \\
$V_{1} P_{y}$ & 0 & 0 & 0 & 0 & 0 & 0 & 0 & 0 & 0 \\
$V_{1} P_{z}$ & 0 & 0 & 0 & 0 & 0 & 0 & 0 & 0 & 0 \\
$V_{2} P_{x}$ & -1 & -0.9673 & -0.8108 & -0.5938 & -0.3851 & -0.2182 & -0.1005 & -0.0295 & 0 \\
$V_{2} P_{y}$ & 0 & 0.0327 & 0.1892 & 0.4062 & 0.6149 & 0.7818 & 0.8995 & 0.9705 & 1 \\
$V_{2} P_{z}$ & 0 & -0.2513 & -0.5539 & -0.6946 & -0.6882 & -0.5841 & -0.4252 & -0.2391 & 0 \\
$V_{3} P_{x}$ & 0 & 0.0327 & 0.1892 & 0.4062 & 0.6149 & 0.7818 & 0.8995 & 0.9705 & 1 \\
$V_{3} P_{y}$ & 0 & 0.0327 & 0.1892 & 0.4062 & 0.6149 & 0.7818 & 0.8995 & 0.9705 & 1 \\
$V_{3} P_{z}$ & 0 & -0.2513 & -0.554 & -0.6945 & -0.6882 & -0.5841 & -0.4252 & -0.2391 & 0 \\
$V_{4} P_{x}$ & 1 & 1 & 1 & 1 & 1 & 1 & 1 & 1 & 1 \\
$V_{4} P_{y}$ & 0 & 0 & 0 & 0 & 0 & 0 & 0 & 0 & 0 \\
$V_{4} P_{z}$ & 0 & 0 & 0 & 0 & 0 & 0 & 0 & 0 & 0 \\
$V_{5} P_{x}$ & 0 & 0 & 0 & 0 & 0 & 0 & 0 & 0 & 0 \\
$V_{5} P_{y}$ & 0 & 0 & 0 & 0 & 0 & 0 & 0 & 0 & 0 \\
$V_{5} P_{z}$ & 0 & 0 & 0 & 0 & 0 & 0 & 0 & 0 & 0 \\
\hline
\end{tabular}


ASME Journal of Mechanical Design

Table 4 The folding angles of Deployable cylinder

\begin{tabular}{|c|c|c|c|c|c|c|c|c|c|}
\hline $\begin{array}{c}V_{1} t_{1}( \\
\text { Mountain) } \\
\end{array}$ & $\pi$ & 3.4 & 3.8 & 4.2 & 4.6 & 5 & 5.4 & 5.8 & $2 \pi$ \\
\hline $\begin{array}{c}V_{1} t_{2} \text { (Valley } \\
\text { ) }\end{array}$ & 3.1416 & 2.7782 & 2.2415 & 1.7595 & 1.339 & 0.972 & 0.6452 & 0.345 & 0 \\
\hline $\begin{array}{c}V_{1} t_{3}( \\
\text { Mountain) }\end{array}$ & 6.2832 & 6.2832 & 6.2832 & 6.2832 & 6.2832 & 6.2832 & 6.2832 & 6.2832 & 6.2832 \\
\hline $\begin{array}{c}V_{2} t_{2}(\text { Valley } \\
\text { ) }\end{array}$ & 3.1416 & 2.7782 & 2.2414 & 1.7595 & 1.339 & 0.9719 & 0.6452 & 0.345 & 0 \\
\hline $\begin{array}{c}V_{3} t_{2}( \\
\text { Mountain) }\end{array}$ & 3.1416 & 3.505 & 4.0417 & 4.5237 & 4.9442 & 5.3112 & 5.638 & 5.9382 & 6.2832 \\
\hline $\begin{array}{c}V_{4} t_{2}( \\
\text { Mountain) }\end{array}$ & 3.1416 & 3.505 & 4.0417 & 4.5237 & 4.9442 & 5.3112 & 5.638 & 5.9382 & 6.2832 \\
\hline $\begin{array}{c}V_{5} t_{2}(\text { Valley } \\
\text { ) }\end{array}$ & 3.1416 & 2.7782 & 2.2415 & 1.7595 & 1.339 & 0.972 & 0.6452 & 0.345 & 0 \\
\hline $\begin{array}{c}V_{6} t_{2}( \\
\text { Mountain })\end{array}$ & 6.2832 & 6.2832 & 6.2832 & 6.2832 & 6.2832 & 6.2832 & 6.2832 & 6.2832 & 6.2832 \\
\hline $\begin{array}{c}V_{6} t_{3} \text { ( Valley } \\
\text { ) }\end{array}$ & 3.1416 & 2.7782 & 2.2415 & 1.7595 & 1.339 & 0.972 & 0.6452 & 0.345 & 0 \\
\hline $\begin{array}{c}V_{7} t_{2}( \\
\text { Mountain })\end{array}$ & 3.1416 & 3.505 & 4.0417 & 4.5237 & 4.9442 & 5.3112 & 5.638 & 5.9382 & 6.2832 \\
\hline $\begin{array}{c}V_{8} t_{2}( \\
\text { Mountain) }\end{array}$ & 3.1416 & 3.505 & 4.0417 & 4.5237 & 4.9442 & 5.3112 & 5.638 & 5.9382 & 6.2832 \\
\hline $\begin{array}{c}V_{8} t_{3}( \\
\text { Mountain) }\end{array}$ & 3.1416 & 3.4 & 3.8 & 4.2 & 4.6 & 5 & 5.4 & 5.8 & 6.2832 \\
\hline
\end{tabular}


ASME Journal of Mechanical Design

Table 5 The coordinates changes of the vertices

\begin{tabular}{|c|c|c|c|c|c|c|c|c|c|}
\hline$V_{1} t_{1}(\mathrm{rad})$ & $\pi$ & 3.4 & 3.8 & 4.2 & 4.6 & 5 & 5.4 & 5.8 & $2 \pi$ \\
\hline$V_{1} P_{x}$ & 0 & 0 & 0 & 0 & 0 & 0 & 0 & 0 & 0 \\
\hline$V_{1} P_{y}$ & 0 & 0 & 0 & 0 & 0 & 0 & 0 & 0 & 0 \\
\hline$V_{1} P_{z}$ & 0 & 0 & 0 & 0 & 0 & 0 & 0 & 0 & 0 \\
\hline$V_{2} P_{x}$ & 1 & 0.9674 & 0.8108 & 0.5938 & 0.3851 & 0.2182 & 0.1005 & 0.0295 & 0 \\
\hline$V_{2} P_{y}$ & 0 & 0.0326 & 0.1892 & 0.4062 & 0.6149 & 0.7818 & 0.8995 & 0.9705 & 1 \\
\hline$V_{2} P_{z}$ & 0 & -0.2513 & -0.5539 & -0.6946 & -0.6882 & -0.5841 & -0.4252 & -0.2392 & 0 \\
\hline$V_{3} P_{x}$ & 3 & 2.7104 & 1.4401 & 0.0042 & -1.0215 & -1.5914 & -1.8591 & -1.9671 & -2 \\
\hline$V_{3} P_{y}$ & 0 & 0.159 & 0.8029 & 1.371 & 1.5621 & 1.464 & 1.2611 & 1.0849 & 1 \\
\hline$V_{3} P_{z}$ & 0 & -1.2238 & -2.3504 & -2.3442 & -1.7484 & -1.0939 & -0.5962 & -0.2673 & 0 \\
\hline$V_{4} P_{x}$ & 5 & 4.6451 & 3.0615 & 1.1918 & -0.2512 & -1.1551 & -1.658 & -1.9081 & -2 \\
\hline$V_{4} P_{y}$ & 0 & 0.2243 & 1.1814 & 2.1834 & 2.7918 & 3.0277 & 3.0601 & 3.026 & 3 \\
\hline$V_{4} P_{z}$ & 0 & -1.7265 & -3.4584 & -3.7333 & -3.1248 & -2.262 & -1.4467 & -0.7457 & 0 \\
\hline$V_{5} P_{x}$ & 7 & 6.6451 & 5.0615 & 3.1918 & 1.7488 & 0.8449 & 0.342 & 0.0919 & 0 \\
\hline$V_{5} P_{y}$ & 0 & 0.2243 & 1.1815 & 2.1833 & 2.7917 & 3.0278 & 3.0601 & 3.026 & 3 \\
\hline$V_{5} P_{z}$ & 0 & -1.7264 & -3.4584 & -3.7333 & -3.1248 & -2.2619 & -1.4467 & -0.7457 & 0 \\
\hline$V_{6} P_{x}$ & 8 & 7.6125 & 5.8723 & 3.7856 & 2.134 & 1.063 & 0.4425 & 0.1213 & 0 \\
\hline$V_{6} P_{y}$ & 0 & 0.2569 & 1.3708 & 2.5895 & 3.4066 & 3.8097 & 3.9595 & 3.9965 & 4 \\
\hline$V_{6} P_{z}$ & 0 & -1.9777 & -4.0124 & -4.4279 & -3.813 & -2.846 & -1.8719 & -0.9848 & 0 \\
\hline$V_{7} P_{x}$ & 6 & 5.8694 & 5.2429 & 4.3752 & 3.5406 & 2.8726 & 2.4021 & 2.1179 & 2 \\
\hline$V_{7} P_{y}$ & 0 & 0.1306 & 0.7571 & 1.6247 & 2.4593 & 3.1275 & 3.5979 & 3.8821 & 4 \\
\hline$V_{7} P_{z}$ & 0 & -1.0053 & -2.2159 & -2.7781 & -2.7527 & -2.3361 & -1.701 & -0.9567 & 0 \\
\hline$V_{8} P_{x}$ & 2 & 2 & 2 & 2 & 2 & 2 & 2 & 2 & 2 \\
\hline$V_{8} P_{y}$ & 0 & 0 & 0 & 0 & 0 & 0 & 0 & 0 & 0 \\
\hline$V_{8} P_{z}$ & 0 & 0 & 0 & 0 & 0 & 0 & 0 & 0 & 0 \\
\hline$V_{9} P_{x}$ & 0 & 0 & 0 & 0 & 0 & 0 & 0 & 0 & 0 \\
\hline$V_{9} P_{y}$ & 0 & 0 & 0 & 0 & 0 & 0 & 0 & 0 & 0 \\
\hline$V_{9} P_{z}$ & 0 & 0 & 0 & 0 & 0 & 0 & 0 & 0 & 0 \\
\hline
\end{tabular}

